\title{
ON SPRINGER REPRESENTATIONS AND THE ZUCKERMAN FUNCTOR
}

\author{
SAM EvENS
}

The purpose of this note is to resolve some questions raised by Hunziker and Wallach. Wallach has given a beautiful, simple algebraic approach to the Springer representations. Hunziker and Wallach have given a conjectural reinterpretation of this result which depends on a computation of the Zuckerman functor. We do this calculation using the geometric interpretation of the Zuckerman functor due to Bernstein, which allows us to deduce the computation from a result of Hotta and Kashiwara. As a consequence we give an explicit connection between Wallach's construction of Weyl group representations and a construction due to Hotta and Kashiwara.

Since this note is rather short, we postpone notation and the statement of the main theorem (Theorem 1.2) to Section 1, where we also reduce 1.2 to a statement relating the Zuckerman and Bernstein induction functors. In section two, we verify this statement by using theorems of Beilinson, Bernstein-Lunts, and Pandzić [BL2, Pa]. We would like to thank Hunziker and Wallach for enlightening and encouraging conversations, Lunts for sending a preprint of [BL2], and Milicić for informing me about Pandzić's thesis. We would also like to thank the referee for correcting an error in Section 1.9. In addition, we thank the University of California, San Diego for hospitality during part of the preparation of this paper.

\section{Holonomicity and vanishing of certain induced modules.}

1.1. Notation. Let $G$ be a complex semisimple group with Borel subgroup $B$ and Cartan subgroup $H$ contained in $B$. Denote Lie algebras of Lie groups by german letters $\mathfrak{g}, \mathfrak{b}, \mathfrak{h}$, etc. Let $W$ be the Weyl group of $G$ with respect to $H$ and let $D(\mathfrak{g})$ and $D(\mathfrak{b})$ denote the algebraic differential operators on $\mathfrak{g}$ and $\mathfrak{b}$ respectively. The polynomial functions $R(\mathfrak{b})$ on $\mathfrak{b}$ form an irreducible $D(\mathfrak{b})$ module. The inclusion $i: \mathfrak{b} \hookrightarrow \mathfrak{g}$ induces by direct image a $D(\mathfrak{g})$ module $M=i_{+}(R(\mathfrak{b})) . M$ is irreducible and holonomic, as follows from Kashiwara's theorem [Bo, 7.11] since $R(\mathfrak{b})$ is irreducible and holonomic. Notice also that since $R(\mathfrak{b})$ is an $H$-module under the adjoint action and $i$ is $H$-equivariant, it follows that $M$ is $H$-equivariant. Then $M$ is a compatible $(D(\mathfrak{g}), H)$ module 
in the sense that the differential of the $H$-action coincides with the induced action of $\mathfrak{h}$ as a Lie subalgebra of $D(\mathfrak{g})$ under the adjoint action. Embed $\mathfrak{g}$ inside $D(\mathfrak{g})$ using the adjoint action. This makes $M$ into a $(\mathfrak{g}, H)$ module. Let $\Gamma^{i}=\Gamma_{H}^{G}: M(\mathfrak{g}, H) \rightarrow M(\mathfrak{g}, G)$ denote the ith Zuckerman functor from $(\mathfrak{g}, H)$ modules to $(\mathfrak{g}, G)$ modules. $\Gamma^{i}(M)$ is still a $D(\mathfrak{g})$ module $([\mathbf{H W}, 5.2])$.

We denote the sheaf of functions on a variety $X$ by $\mathcal{O}_{X}$ and for a map $f: Y \rightarrow X, f^{o}: M\left(\mathcal{O}_{X}\right) \rightarrow M\left(\mathcal{O}_{Y}\right)$ is the usual pullback in the category of quasicoherent sheaves or its derived category extension. We denote the dimension of $X$ by $d_{X}$. If $C$ is a complex, then $C \cdot[s]$ denotes the shift of the complex $C \cdot$ by $s$.

Theorem 1.2. Let $r=\operatorname{dim} \mathfrak{g} / \mathfrak{b}$. Then $\Gamma^{i}(M)=0$ if $i \neq r$ and $\Gamma^{r}(M)$ is holonomic.

Remark 1.3. $\quad M$ can be identified with the $D(\mathfrak{g})$ module denoted by $M$ in $[\mathbf{H W}]$ by an easy calculation. Hunziker and Wallach proved Theorem 1.2 under the hypothesis that $M$ is free for the opposite nilradical of $\mathfrak{b}$ (see [HW], 8.2 and 8.5). We prove 1.2 independently of this hypothesis. The proof will be given in the remainder of section one.

1.4. The equivariant derived category and the geometric induction functor ([BL1], $[\mathrm{BL} 2],[\mathrm{MV}])$. Let $X$ be a variety and $A$ an algebraic group acting on $X$. Denote by $\mathcal{D}_{X}$ the sheaf of algebraic differential operators on $X$ and let $D_{A}(X)$ be the equivariant derived category of $\mathcal{D}_{X}$ modules. Recall that a compatible $\left(\mathcal{D}_{X}, A\right)$ module (meaning as before that the differential of the $A$ action coincides with the action of $\mathfrak{a}$ induced from the mapping $\mathfrak{a} \rightarrow$ vector fields on $X)$ may be regarded as on object in $D_{A}(X)$ concentrated in degree 0 . For an equivariant map $f: Y \rightarrow X$ the usual functors $f^{+}, f^{!}, f_{+}, f_{\text {! }}$ extend to the equivariant derived category. Moreover, if $N \subset A$ is a normal subgroup acting freely on $X$ then the quotient map $q: X \rightarrow X / N$ induces an equivalence $q^{+}: D_{A / N}(X / N) \rightarrow D_{A}(X)$. Let $B \subset A$ be a subgroup and consider the diagram

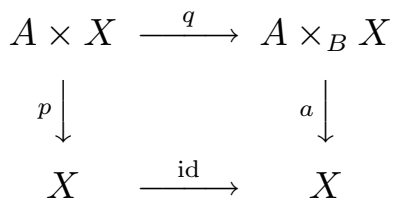

where $p$ is projection onto the second factor, $q$ is the obvious quotient map, and $a$ is the action morphism. Here $B$ acts on $A \times X$ by $b \cdot(y, x)=\left(y b^{-1}, b x\right)$. Then we have a geometric induction functor $\gamma_{B}^{A}: D_{B}(X) \rightarrow D_{A}(X)$ given by $\gamma_{B}^{A}(\mathcal{F})=a_{+}(\mathcal{S})$, where $\mathcal{S}$ is the unique object in $D_{A}\left(A \times_{B} X\right)$ such 
that $q^{+}(\mathcal{S})=p^{+}(\mathcal{F})$. Then $\gamma$ is right adjoint to the forgetful functor, i.e., $R \operatorname{Hom}_{D_{B}(X)}(\mathcal{M}, \mathcal{N})=R \operatorname{Hom}_{D_{A}(X)}\left(\mathcal{M}, \gamma_{B}^{A}(\mathcal{N})\right)$ (see [MV], e.g.).

Proposition 1.5. Let $H \subset K$ be reductive subgroups of $G$ and let $M$ be a compatible $(D(\mathfrak{g}), H)$ module, which is by restriction a $(\mathfrak{g}, H)$ module and also may be regarded as an object in $D_{H}(\mathfrak{g})$. Then $\Gamma_{H}^{K^{i}}(M) \cong H^{i}\left(\gamma_{H}^{K}(M)\right)$ as $K$-modules.

We will prove this theorem in Section 2.

We return to the proof of Theorem 1.2. We must compute $\gamma_{H}^{G}(M)$. Observe that $M$ is equivariant for the Borel subgroup $B$ and may be regarded as an object in $D_{B}(\mathfrak{g})$.

Lemma 1.6. Let $M \in D_{B}(\mathfrak{g})$. Then $\gamma_{B}^{G}(M)=\gamma_{H}^{G}(M)$.

Proof. Consider the diagram

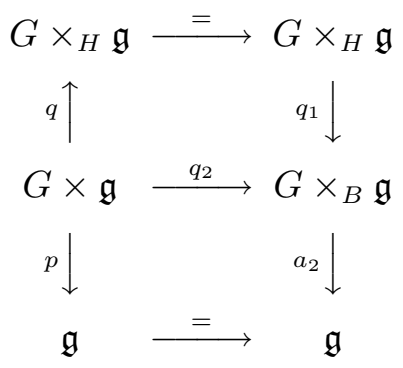

where $q, q_{1}$, and $q_{2}$ are quotient maps and $a_{2}(g, X)=g \cdot X$, so $a=a_{2} \circ q_{1}$. Then $\gamma_{H}^{G}(M)=a_{+}(\mathcal{S})$, where $q^{+}(\mathcal{S})=p^{+}(M)$. But $q_{2}{ }^{+}\left(\mathcal{S}_{2}\right)=q^{+} q_{1}{ }^{+}\left(\mathcal{S}_{2}\right)$, so we may take $\mathcal{S}=q_{1}{ }^{+}\left(\mathcal{S}_{2}\right)$, where $q_{2}{ }^{+}\left(\mathcal{S}_{2}\right)=p^{+}(M)$. Then $\gamma_{H}^{G}(M)=$ $a_{+}\left(q_{1}{ }^{+}\left(\mathcal{S}_{2}\right)\right)=a_{2+}\left(q_{1+} q_{1}{ }^{+}\left(\mathcal{S}_{2}\right)\right)$. But $q_{1_{+}} q_{1}{ }^{+}$is the identity functor. To see this, let $D R$ be the de Rham functor and observe that it suffices to prove $D R q_{1_{+}} q_{1}{ }^{+} \mathcal{F}=D R \mathcal{F}$ for any $\mathcal{D}_{G \times_{B} \mathfrak{g}}$ complex $\mathcal{F}$ with regular singularities, or equivalently $q_{1 *} q_{1}{ }^{*} \mathcal{F}=\mathcal{F}$ for any constructible sheaf. This follows from the projection formula and the fact that $q_{1}$ is a fiber bundle with vector space fibers. Thus, $\gamma_{H}^{G}(M)=a_{2+}\left(\mathcal{S}_{2}\right)=\gamma_{B}^{G}(M)$.

1.7. (See $[\mathrm{MV}],[\mathrm{Mi}]$ and others for analogous calculations.) Consider the Cartesian diagram

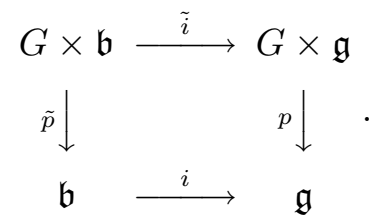

We will replace $M$ by its localization, the $\mathcal{D}_{\mathfrak{g}}$ module $\mathcal{M}=i_{+}\left(\mathcal{O}_{\mathfrak{b}}\right)$. Since $\tilde{p}$ is smooth, $p^{+}(\mathcal{M})=\tilde{i}_{+} \tilde{p}^{+}\left(\mathcal{O}_{\mathfrak{b}}\right)$ by base change and $\tilde{p}^{+}\left(\mathcal{O}_{\mathfrak{b}}\right)=\mathcal{O}_{G \times \mathfrak{b}}\left[-d_{G}\right]$. 
Let $\hat{q}: G \times \mathfrak{b} \rightarrow G \times \times_{B} \mathfrak{b}$ and $\hat{i}: G \times_{B} \mathfrak{b} \hookrightarrow G \times_{B} \mathfrak{g}$ be the obvious quotient and inclusion. Then

$$
\mathcal{O}_{G \times \mathfrak{b}}=\hat{q}^{0}\left(\mathcal{O}_{G \times{ }_{B} \mathfrak{b}}\right)=\hat{q}^{+}\left(\mathcal{O}_{G \times{ }_{B} \mathfrak{b}}\right)\left[d_{B}\right],
$$

SO

$$
p^{+} i_{+} \mathcal{O}_{\mathfrak{b}}=\tilde{i}_{+} \hat{q}^{+} \mathcal{O}_{G \times_{B} \mathfrak{b}}\left[-d_{G / B}\right]=q_{2}{ }^{+} \hat{i}_{+} \mathcal{O}_{G \times_{B} \mathfrak{b}}\left[-d_{G / B}\right] .
$$

Since we have $a_{2+} \hat{i}_{+}=f_{+}$where $f: G \times{ }_{B} \mathfrak{b} \rightarrow \mathfrak{g}$ is the action map, we obtain $\gamma_{B}^{G}\left(i_{+} \mathcal{O}_{\mathfrak{b}}\right)=a_{2+} \hat{i}_{+} \mathcal{O}_{G \times_{B} \mathfrak{b}}\left[-d_{G / B}\right]=f_{+} \mathcal{O}_{G \times_{B} \mathfrak{b}}[-r]$. Then using Lemma 1.6, we obtain

$$
H^{i}\left(\gamma_{H}^{G}\left(i_{+} \mathcal{O}_{\mathfrak{b}}\right)\right)=H^{i-r}\left(f_{+} \mathcal{O}_{G \times_{B} \mathfrak{b}}\right) .
$$

Theorem $1.8[\mathbf{H K}, 4.2 .3] . \quad H^{0} f_{+}\left(\mathcal{O}_{G \times_{B} \mathfrak{b}}\right)$ is a holonomic $\mathcal{D}_{\mathfrak{g}}$ module equal to the minimal extension of its restriction to the regular set and $H^{i} f_{+}\left(\mathcal{O}_{G \times_{B} \mathfrak{b}}\right)$ vanishes for $i \neq 0$.

Hotta and Kashiwara do their calculation in $D(\mathfrak{g})$, but it is easy to check that their argument is also valid in $D_{G}(\mathfrak{g})$.

The proof of 1.2 now follows from 1.5, 1.7.1, and 1.8.

1.9. Denote the $D(\mathfrak{g})$ module $\Gamma^{r}(M)$ by $N . N$ decomposes into a direct sum $N=\oplus_{\chi \in \hat{W}} U^{\chi} \otimes N^{\chi}$, where the $U^{\chi}$ are pairwise inequivalent irreducible $W$-modules and the $N^{\chi}$ are pairwise inequivalent irreducible $D(\mathfrak{g})$ modules [HK, 5.4.2]. For a $G$ module $A$, denote $G$ invariants by $A^{G}$. There is a Harish-Chandra homomorphism $\delta: D(\mathfrak{g})^{G} \rightarrow D(\mathfrak{h})^{W}$ (see [HW]). Moreover, $R(\mathfrak{h})$ is a $D(\mathfrak{h})$ module using the obvious action, and $R(\mathfrak{h})$ also has a $W$ action given by the $W$ action on $\mathfrak{h}$. In $[\mathbf{W a}]$, it is shown that as a $(D(\mathfrak{h}), W)$ module, $R(\mathfrak{h}) \cong U^{\chi} \otimes V^{\chi}$, where the $V^{\chi}$ are pairwise inequivalent $D(\mathfrak{h})^{W}$ modules. It follows that $\delta^{*} R(\mathfrak{h}) \cong \oplus U^{\chi} \otimes \delta^{*} V^{\chi}$. We wish to relate the modules $N^{\chi}$ to the $\delta^{*} V^{\chi}$.

In Section 7 of $\left[\mathbf{H W}\right.$, it is shown that as $D(\mathfrak{g})^{G}$ modules, $N^{G} \cong \delta^{*} R(\mathfrak{h})$. We claim that as $\left(D(\mathfrak{g})^{G}, W\right)$ modules, $N^{G} \cong \delta^{*} R(\mathfrak{h}) \otimes s g n$, so that $N^{G}$ differs from $\delta^{*} R(\mathfrak{h})$ by the sign involution (this observation and the ensuing argument are essentially due to the referee). The claim can be established by comparing the two modules on the set of regular semisimple elements $\mathfrak{g}_{\text {reg }}$, since $N$ is given by extending $\left.N\right|_{\mathfrak{g}_{\text {reg }}}$ in a canonical way [Lu] and the functor $\operatorname{Mod}\left(D(\mathfrak{g})^{G}\right) \rightarrow \operatorname{Mod}(D(\mathfrak{g}))$ given by tensoring takes irreducibles to irreducibles $[\mathbf{H W}]$. We can identify $\left.N\right|_{\mathfrak{g}_{\text {reg }}}=\mathcal{O}\left(G \times_{H} \mathfrak{h}_{\text {reg }}\right)$, where $\mathfrak{h}_{\text {reg }}$ is the set of elements in $\mathfrak{h}$ not perpindicular to any root. It follows that $\left(\left.N\right|_{\mathfrak{g}_{\mathrm{reg}}}\right)^{G} \cong R\left(\mathfrak{h}_{\mathrm{reg}}\right)$. This is an isomorphism of $D\left(\mathfrak{g}_{\mathrm{reg}}\right)^{G}$ modules where the $D\left(\mathfrak{g}_{\text {reg }}\right)^{G}$ module structure on $R\left(\mathfrak{h}_{\text {reg }}\right)$ is given by $D \cdot\left(\left.f\right|_{\mathfrak{h}_{\text {reg }}}\right)=\left.D(f)\right|_{\mathfrak{h}_{\text {reg }}}$. The $D\left(\mathfrak{g}_{\text {reg }}\right)$ structure used in $[\mathbf{H W}]$ on $R\left(\mathfrak{h}_{\text {reg }}\right)$ is the conjugation of the above 
structure by $\pi$, the product of all positive roots with respect to an ordering. It follows that the map $\left(\left.N\right|_{\mathfrak{g}_{\text {reg }}}\right)^{G} \rightarrow R\left(\mathfrak{h}_{\text {reg }}\right) \otimes \operatorname{sgn}$ given by $f \mapsto f \cdot \pi$ is an isomorphism of $\left(D\left(\mathfrak{g}_{\mathrm{reg}}\right)^{G}, W\right)$ modules.

Since the $G$ action on $N$ commutes with the $W$ action, it follows that $N^{G} \cong \oplus_{\chi \in \hat{W}} U^{\chi} \otimes\left(N^{\chi}\right)^{G}$. The claim of the previous paragraph implies that $\left(N^{\chi}\right)^{G} \cong V^{\chi \otimes s g n}$. This amounts to Theorem 8.8 in $[\mathbf{H W}]$, which was proved there under the freeness assumption discussed in 1.3.

Moreover, we obtain an explicit connection between the classification of Weyl group representations given in $[\mathbf{W a}]$ and the classification of Weyl group representations implicit in $[\mathbf{H K}]$. Indeed, the Fourier transform $F_{\mathfrak{g}}(N)=\oplus U^{\chi} \otimes F_{\mathfrak{g}}\left(N^{\chi}\right)$. Each $F_{\mathfrak{g}}\left(N^{\chi}\right)$ is an irreducible $D(\mathfrak{g})$-module supported on the nilpotent cone $([\mathbf{H K}, 5.3])$, so is determined by a nilpotent orbit with a local system. Thus, we have proved the following result, which is essentially proven for trivial local systems in [Wa].

Theorem 1.10. The Weyl group representation corresponding to the Dmodule $F_{\mathfrak{g}}\left(N^{\chi}\right)$ in the Hotta-Kashiwara picture corresponds to $V^{\chi \otimes s g n}$ in Wallach's description of the Springer representations.

\section{Compatability of the Zuckerman functor with geometric induction.}

In this section, we prove Proposition 1.5 by using results of Beilinson, Bernstein-Lunts, and Pandzić. Presumably a less technical proof can be given by explicit calculation of the Bernstein functor.

2.1. The homotopy category of equivariant modules. This section is a summary of results from [BL2]. Let $(A, K)$ be a Harish-Chandra pair. This means that $A$ is an associative $\mathbb{C}$ algebra with 1 and $K$ is an algebraic group acting on $A$ by $\sigma: K \rightarrow \operatorname{Aut}(A)$ and we are also given an embedding $j: \mathfrak{k} \hookrightarrow A$ satisfying,

(a) $j$ is $K$-equivariant,

(b) $d \sigma(X)(a)=[j(x), a]$ for $X \in \mathfrak{k}$ and $a \in A$.

A weak $(A, K)$-module $M$ is a vector space with an algebraic representation $\rho: K \rightarrow \operatorname{Aut}(M)$ and an algebra representation $\alpha: A \rightarrow \operatorname{End}(M)$ such that for $a \in A$ and $k \in K$, we have $\alpha(\sigma(k)(a))=\rho(k) \alpha(a) \rho(k)^{-1}$. $M$ is called an $(A, K)$ module if $d \rho=\alpha \circ j$ on $\mathfrak{k}$. An $h$-complex is a complex $C$. of weak $(A, K)$-modules together with a linear map $i: \mathfrak{k} \rightarrow \operatorname{Hom}^{-1}\left(C^{\cdot}, C^{\cdot}\right)$ such that

(a) $\rho(k) i(X) \rho(k)^{-1}=i(\operatorname{Ad}(k)(X))$, for $X \in \mathfrak{k}, k \in K$

(b) $i(\mathfrak{k})$ commutes with the $A$ action

(c) $i(X) i(Y)+i(Y) i(X)=0$ for $X, Y \in \mathfrak{k}$ 
(d) $d i(X)+i(X) d=d \rho(X)-\alpha(j(X))$, for $X \in \mathfrak{k}$.

It follows immediately that $H^{i}\left(C^{\cdot}\right)$ is an $(A, K)$-module. Let $C_{h}(A, K)$ be the abelian category of $h$-complexes and let $D_{h}^{b}(A, K)$ be the corresponding derived category of bound-ed complexes. All of these constructions are local, so we may consider the sheaf of Harish-Chandra pairs and the associated derived category of modules, $D_{h}^{b}\left(\mathcal{D}_{X}, K\right)$. The following theorem of Beilinson explains why we have introduced this category.

Theorem 2.2 [BL2, 2.13]. Let $X$ be a smooth $K$-variety. There is an equivalence of categories $D_{h}^{b}\left(\mathcal{D}_{X}, K\right) \cong D_{K}^{b}(X)$. For a $\left(\mathcal{D}_{X}, K\right)$ module $\mathcal{M}$, this equivalence is given by the obvious identification.

Since the global section functor induces an equivalence $D_{h}^{b}\left(\mathcal{D}_{\mathfrak{g}}, K\right) \cong$ $D_{h}^{b}(D(\mathfrak{g}), K)$, we obtain the following corollary.

Corollary 2.3. Let $K \subset G$ be a subgroup. Then $D_{K}^{b}(\mathfrak{g}) \cong D_{h}^{b}(D(\mathfrak{g}), K)$.

2.4. Induction in $D_{h}^{b}(A, K)[\mathrm{Pa}]$. Assume $K$ is reductive and let $H \subset K$ be a reductive subgroup. Then $(A, H)$ is also a Harish-Chandra pair.

Theorem $2.5[\mathrm{~Pa}]$. There is an algebraic induction functor $\Gamma_{H}^{K}$ : From $D_{h}^{b}(A, H)$ to $D_{h}^{b}(A, K)$ right adjoint to the forgetful functor. Suppose $B$ is a subalgebra of $A$ such that $(B, K)$ and $(B, H)$ are Harish-Chandra pairs and $A$ is flat over $B$. Then induction commutes with the natural restriction maps $D_{h}^{b}(A, H) \rightarrow D_{h}^{b}(B, H)$ and $D_{h}^{b}(A, K) \rightarrow D_{h}^{b}(B, K)$.

We briefly describe the construction of the induction functor. It is defined as the derived functor of an induction functor $C_{h}^{b}(A, H) \rightarrow C_{h}^{b}(A, K)$. Recall that $C_{h}^{b}(A, H)$ is equivalent to the category of differential graded modules for a differential graded algebra [Gi, BL2]. Indeed, consider the differential graded Lie algebra $\overline{\mathfrak{h}}$, where $\overline{\mathfrak{h}}^{0}=\mathfrak{h}$ as a Lie algebra, $\overline{\mathfrak{h}}^{-1}=\mathfrak{h}$ as a vector space and has zero bracket, $\overline{\mathfrak{h}}^{i}=0$ for $i \neq 0,-1$. The differential $d: \overline{\mathfrak{h}}^{-1} \rightarrow \overline{\mathfrak{h}}^{0}$ is the identity map and for $X \in \overline{\mathfrak{h}}^{0}=\mathfrak{h}, Y \in \overline{\mathfrak{h}}^{-1}$, we have $d[X, Y]=[X, d Y]$. Then $U(\overline{\mathfrak{h}}) \otimes A$ is a differential graded algebra and the group $H$ acts by conjugation on $U(\overline{\mathfrak{h}}) \otimes A$. The diagonal embedding $\mathfrak{h} \rightarrow B_{H}, X \mapsto X \otimes 1+1 \otimes j(X)$ makes $\left(B_{H}, H\right)$ into a differential graded Harish-Chandra pair. Then we have an equivalence of categories between $C_{h}(A, H)$ and the category of differential graded Harish-Chandra modules $M\left(B_{H}, H\right)$ [BL2, 1.11.1]. Note that $(U(\mathfrak{k}) \otimes U(\overline{\mathfrak{h}}), H)$ is also a differential graded Harish-Chandra pair in an analogous way. Moreover, $U(\overline{\mathfrak{k}})$ is a Harish-Chandra module for this algebra. For a module $V \in C_{h}^{b}(A, H)$, then $R(K) \otimes V$ is in a natural way a $(U(\mathfrak{k}) \otimes U(\overline{\mathfrak{h}}), H)$ module, which also has a commuting $(A, K)$ action. Then 
$\Gamma_{H}^{K}(V)=\operatorname{Hom}_{U(\mathfrak{k}) \otimes U(\overline{\mathfrak{h}}), H}(U(\overline{\mathfrak{k}}), R(K) \otimes V)$ is a $(A \otimes U(\overline{\mathfrak{k}}), K)$ module in a natural way. We use $\Gamma_{H}^{K}$ to denote the corresponding derived functor.

Lemma 2.6. The following diagram commutes.

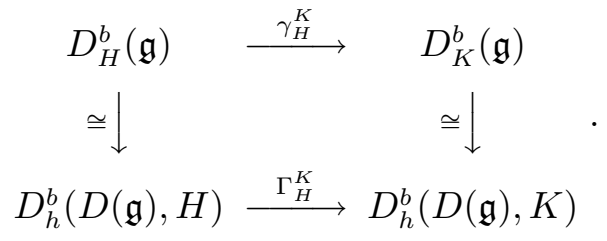

Proof. The commutativity of the diagram

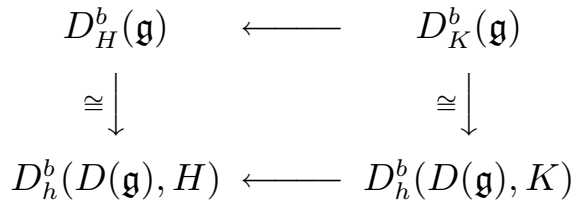

where the horizontal arrows are forgetful functors is obvious. The lemma follows from the uniqueness of adjoint functors.

Let $A=U(\mathfrak{g}) \otimes_{\pi} D(\mathfrak{g})$, where $U(\mathfrak{g})$ acts on $D(\mathfrak{g})$ by extending the adjoint action (a similar construction is given in $[\mathbf{H W}, 5.2]$ ). It is not hard to check that $A$ is free over $U(\mathfrak{g})$ and $D(\mathfrak{g})$ by using obvious filtrations.

Lemma 2.7. The following diagrams are commutative, where the vertical arrows are given by restriction.

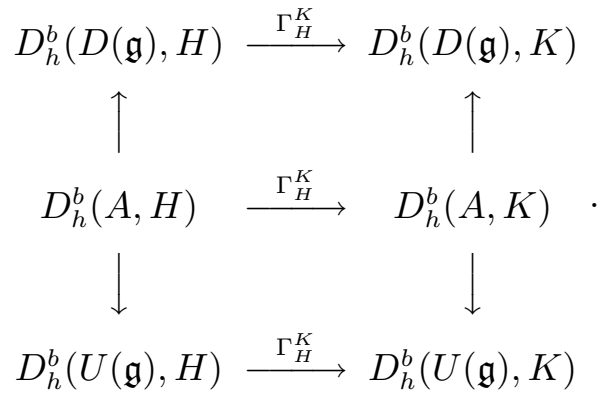

Proof. This follows from 2.5.

We recall one of the main theorems from [BL2].

Theorem 2.8 [BL2, 1.3]. The natural functor $\alpha$ from $D^{b}(U(\mathfrak{g}), K)$ to $D_{h}^{b}(U(\mathfrak{g}), K)$ is an equivalence of categories.

Since the forgetful functor commutes with $\alpha$, it follows immediately that the algebraic induction functor $\Gamma_{H}^{K}$ corresponds to the usual Zuckerman functor under the equivalence $\alpha$. 
2.9. Proof of 1.5. Regard $M$ as an object in $D_{h}^{b}(A, H)$ by making $U(\mathfrak{g})$ act by the restriction of the $D(\mathfrak{g})$ action. The proof follows from Lemma 2.6, Lemma 2.7, and Theorem 2.8.

\section{References}

[BL1] J. Bernstein and V. Lunts, Equivariant sheaves and functors, Lecture Notes in Mathematics, 1578, Springer-Verlag, 1994.

[BL2] Localization for derived categories of $(\mathfrak{g}, K)$ modules, J. of AMS, 8 (1995), 819-851.

[Bo] A. Borel, et al., Algebraic D-modules, Academic Press, 1987.

[Gi] V. Ginzburg, Equivariant cohomology and Kahler geometry, Func. Anal. and its Applications, 21 (1987), 19-34.

[HK] R. Hotta and M. Kashiwara, The invariant holonomic system on a semi-simple Lie algebra, Invent. Math., 75 (1984), 327-358.

[HW] M. Hunziker and N. Wallach, On the Harish-Chandra homomorphism of invariant differential operators on a reductive Lie algebra, Representation theory and harmonic analysis, in Contemporary Mathematics, 191 (1995).

[Lu] G. Lusztig, Green polynomials and singularities of unipotent classes, Adv. in Math., 42 (1981), 169-178.

[Mi] I. Mirković, Character sheaves on reductive Lie algebras, preprint.

[MV] I. Mirković and K. Vilonen, Characteristic varieties of character sheaves, Invent. Math., 93 (1988), 405-418.

[Pa] P. Pandzić, Equivariant analogues of Zuckerman functors, Utah thesis, 1995.

[Vo] D. Vogan, Representations of real reductive Lie groups, Birkhauser, 1981.

[Wa] N. Wallach, Invariant differential operators on a reductive Lie algebra and Weyl group representations, J. Amer. Math. Soc., 6 (1993), 779-816.

Received September 21, 1995. The author is partially supported by a NSF postdoctoral fellowship.

UnIVERSity of ARIZONA

TuCsON, AZ 85721

E-mail address: evens@math.arizona.edu.

Note: Theorem 1.10 Appeared in the PAPER Version AS Theorem 1.19. 